\title{
Scapular kinematic and shoulder muscle activity alterations after serratus anterior muscle fatigue
}

\section{$\operatorname{AUTHOR}(S):$}

Umehara, Jun; Kusano, Ken; Nakamura, Masatoshi; Morishita, Katsuyuki; Nishishita, Satoru; Tanaka, Hiroki; Shimizu, Itsuroh; Ichihashi, Noriaki

\section{CITATION:}

Umehara, Jun ... [et al]. Scapular kinematic and shoulder muscle activity alterations after serratus anterior muscle fatigue. Journal of Shoulder and Elbow Surgery 2018, 27: 12051213

\section{ISSUE DATE:}

2018-07

URL:

http://hdl.handle.net/2433/244284

\section{RIGHT:}

(C) 2018. This manuscript version is made available under the CC-BY-NC-ND 4.0 license http://creativecommons.org/licenses/by-nc-nd/4.0/.; This is not the published version. Please cite only the published version.; この論文は出版社版でありません。引用の際には 出版社版をご確認ご利用ください。 
Scapular kinematics and serratus anterior fatigue

\section{Title page}

2 Scapular kinematic and shoulder muscle activity alterations after serratus anterior muscle

3 fatigue

4

5 Jun Umehara, MS, $\left.\mathrm{PT}_{1}\right)^{*}$, Ken Kusano, MS, PT2), Masatoshi Nakamura, $\left.\mathrm{PhD}, \mathrm{PT}_{3}\right)$, Katsuyuki

6 Morishita, PhD, PT1,4), Satoru Nishishita, MS, PT1), Hiroki Tanaka, MS, PT5), Itsuroh

7 Shimizu, MS, PT6), Noriaki Ichihashi, PhD, PT1)

8

9 1) Human Health Sciences, Graduate School of Medicine, Kyoto University, Kyoto, Japan

10 2) ASICS Corporation, Institution of Sports Science, Kobe, Japan

11 3) Institute for Human Movement and Medical Sciences, Niitaga University of Health and

4) Department of Physical Therapy, Faculty of Social Work Studies, Josai International

5) Rehabilitation Unit, Kyoto University Hospital, Kyoto, Japan

6)Department of Physical Therapy, Fukui General Clinic, Fukui, Japan

*Corresponding author: Jun Umehara, Human Health Sciences, Graduate School of

Medicine, Kyoto University, 53 Shogoin-Kawahara-cho, Kyoto 606-8507, Japan 
Scapular kinematics and serratus anterior fatigue

21 Phone: +81 20 -75-751-3935; Fax: +81-75-751-3909

E-mail: umehara.jun.77z@st.kyoto-u.ac.jp

Financial biases: None

Ethical approval: This study design was approved by the ethics committee of Kyoto

27 University Graduate School and the Faculty of Medicine (R0327). 
Scapular kinematics and serratus anterior fatigue

\section{Abstract}

Background: Although the serratus anterior muscle has an important role in scapular movement, no study to date has investigated the effect of serratus anterior fatigue on scapular kinematics and shoulder muscle activity. The purpose of this study was to clarify the effect of serratus anterior fatigue on scapular movement and shoulder muscle activity.

Methods: The study participants were 16 healthy men participated in this study. Electrical muscle stimulation was used to fatigue the serratus anterior muscle. Shoulder muscle strength and endurance, scapular movement, and muscle activity were measured before and after the fatigue task. The muscle activity of the serratus anterior, upper and lower trapezius, anterior and middle deltoid, and infraspinatus muscles were recorded and the median power frequency of these muscles was calculated to examine the degree of muscle fatigue.

Results: The muscle endurance and median power frequency of the serratus anterior muscle decreased after the fatigue tasks, whereas the muscle activities of the serratus anterior, upper trapezius, and infraspinatus muscles increased. External rotation of the scapula at the shoulder elevated position increased after the fatigue task.

Conclusion: Selective serratus anterior fatigue due to electric muscle stimulation decreased the serratus anterior endurance at the flexed shoulder position. Furthermore, the muscle activities of the serratus anterior, upper trapezius, and infraspinatus increased and the scapular external rotation was greater after serratus anterior fatigue. These results suggest that the rotator cuff 
Scapular kinematics and serratus anterior fatigue

47 and scapular muscle compensated to avoid the increase in internal rotation of the scapula caused

48 by the dysfunction of the serratus anterior muscle.

49 Level of evidence: Basic Science, Kinesiology Study

50 Key words: Shoulder, Scapula, Fatigue, Serratus anterior muscle, Biomechanics,

51 Rehabilitation

52 
Scapular kinematics and serratus anterior fatigue

\section{Introduction}

The shoulder complex consist of the scapula, humerus, and clavicle, and the scapula upwardly and externally rotates and posteriorly tilts during shoulder elevation. ${ }^{28,30}$ This coordinated movement is controlled by the neuromuscular function and capsular ligaments. ${ }^{22}$ Dysfunction of the control system alters the scapular movement and might cause a shoulder disorder. Previous studies reported that the scapular movement during shoulder elevation changed in people with subacromial impingement syndrome, ${ }^{27,29}$ rotator cuff tear, ${ }^{37}$ or shoulder instability..$^{37,41}$

Shoulder muscle activity plays an important role in controlling scapular movement, and the dysfunction of these muscles might be a factor that changes scapular movement. Previous studies found decreased activity of the serratus anterior muscle ${ }^{27}$ and increased activity of the upper trapezius muscle ${ }^{10,27}$ in subacromial impingement syndrome. Furthermore, the serratus anterior and the upper and lower trapezius muscles work as a force couple for upward rotation of the scapula. Decreased activity of the serratus anterior muscle relative to the upper trapezius muscle associated with the change in scapulohumeral rhythm and the decrease in scapular upward rotation during shoulder elevation were found in people with impingement syndrome. ${ }^{19,36}$ These findings in previous studies suggested that the change in muscle activity of the serratus anterior was related to the change in scapular movement. In addition, it was proposed in the consensus statement from the scapular summit also proposed that the serratus 
Scapular kinematics and serratus anterior fatigue

anterior is one of the causes of scapular dyskinesis ${ }^{24}$ and is one of the target muscles for its rehabilitation. ${ }^{12}$ However, the cause-consequence relationship between the dysfunction of the serratus anterior muscle and abnormal scapular movement is unknown.

Some studies investigated the effect of muscle dysfunction caused by acute muscle fatigue on the scapular movement to clarify the relationship between shoulder muscle activity and scapular movement. ${ }^{7,14,32}$ A previous study of serratus anterior fatigue and scapular movement, a previous study examined the effect of a push-up plus task on muscle activity and scapular movement and found an increase in scapular internal rotation and decrease in posterior tilt during scapular plane elevation. However, electromyographically, fatigue of the serratus anterior and upper and lower trapezius and infraspinatus muscles was seen in this study. ${ }^{5}$ Therefore, the relationship between selective fatigue of the serratus anterior and changes in scapular kinematics is unknown, whereas the effect of selective muscle fatigue on scapular kinematics should be elucidated to further develop our knowledge of the shoulder complex. Many previous studies evaluated 3-dimensional scapular motion during only shoulder elevation. However, it is possible that muscle endurance at the shoulder elevated position is important for evaluating shoulder function because some shoulder functional tests apply muscle endurance at the shoulder elevated position, which is impaired in people with shoulder disease. ${ }^{8,39}$ 
Scapular kinematics and serratus anterior fatigue

91 anterior on muscle activity and scapular movement. We hypothesized that scapular upward

92 rotation increased at the shoulder elevated position after the fatigue task due to the

93 compensatory increase in muscle activity of the upper trapezius. 
Scapular kinematics and serratus anterior fatigue

\section{Materials and Methods}

\section{Participants}

This study was a controlled experimental study. The study participants were 16 men (mean age, $25.6 \pm 3.4$ years; mean height, $172.4 \pm 5.4 \mathrm{~cm}$; mean weight, $66 \pm 7.2 \mathrm{~kg}$ ) who were students from our institution. At the time of recruitment, the participants confirmed that they did not meet the exclusion criteria, which included present or history of orthopedic or nervous system disease in the upper limb, athletes, or persons who perform perform any extensive exercise, and female gender. Before the experiment, no participants were excluded. The aim and procedure of this study was explained to all participants, each of whom provided informed consent. The sample size was calculated based on a 2-way analysis of variance (ANOVA) with repeated measures $\left(\right.$ effect size $=0.25$, $\alpha$ error $=0.05$, power $=0.8$ ) using $G^{*}$ Power 3.1 (Heinrich Hein University, Düsseldorf, Germany) before the participants were recruited and showed that a group size of 10 subjects was required for this analysis to enable the detection of statistical significance. Therefore, 16 healthy men were recruited for this study.

\section{Experimental procedures}

The dominant and non-dominant upper limb was identified as the control and fatigue limb, respectively. The participants performed maximal isometric shoulder flexion at $90^{\circ}$ and then kept their arm at shoulder flexion at $90^{\circ}$ to measure the shoulder muscle strength and endurance, 
Scapular kinematics and serratus anterior fatigue

respectively. Scapular kinematics and electromyography (EMG) measurements were collected

during the muscle endurance test. The participants underwent the fatigue task for 25 minutes.

Muscle strength and endurance, scapular movement, and muscle activity were measured again

117 after the fatigue task.

\section{Fatigue task}

120 The fatigue task consisted of electric muscle stimulation of the serratus anterior muscle using musculoskeletal electric stimulator (EU-910, Ito Co. Ltd., Tokyo, Japan) to induce selective muscle fatigue. Participants sat on a stool and skin was shaved and cleaned to reduce the skin resistance. Bipolar electrodes $(2 \mathrm{~cm} \times 2 \mathrm{~cm})$ were attached to the skin over the lower parts of the serratus anterior with tape at level of the sixth rib on the midaxillary line along the leading edge of the latissimus dorsi muscle. The motor point of the lower parts of the serratus anterior muscle was interposed between these electrodes to activate this muscle as much as possible. A

127 high-voltage pulsed current with a $50-\mu$ s pulse width and $100-\mathrm{Hz}$ frequency was used in this study. During the initial 5 minutes of muscle stimulation, the intensity was gradually increased to the maximum level that each subject could tolerate and then sustained for the following 20 minutes. The average voltage intensity was about $30 \mathrm{~V}$. Fatigue induced by electrical muscle

131 stimulation was applied in previous studies and acute loss of muscle strength after electrical 
Scapular kinematics and serratus anterior fatigue

by electrical muscle stimulation using a musculoskeletal electric stimulator.

\section{Muscle strength and endurance}

The muscle strength of shoulder flexion was measured in both upper limbs using the handheld

on a stool performed maximal isometric shoulder flexion for 3 seconds at $90^{\circ}$ shoulder flexion

with the elbow in full extension and the forearm in a neutral position. The shoulder flexion

angle was confirmed by the investigator using the goniometer. The handheld dynamometer was

times with optimal interval. The shoulder flexion strength was expressed as torque, the product 
Scapular kinematics and serratus anterior fatigue

represented as the maximum time that the participant could maintain his posture without deviating while holding the load. The investigator visually confirmed whether the participants could maintain the correct posture and even the slightest lowering of the flexed upper limb, flexion of the elbow, or compensation of the trunk was noted as a deviation from the correct posture. The evaluation of correct posture by the investigator using visual confirmation based on a previous study. ${ }^{33}$ The maximum time for muscle endurance in each upper limb was examined once in a random order with optimal interval.

\section{EMG protocol}

Muscle activity was determined using surface EMG (TeleMyo2400; Noraxon, Scottsdale, AZ, USA) with sampling at $1500 \mathrm{~Hz}$. The skin at the electrode sites was shaved and cleaned using scrubbing gel and alcohol. Disposable pre-gelled Ag-AgCl electrodes (Blue Sensor, Medicotest, Olstykke, Denmark) were placed over the anterior and middle deltoid, upper and lower trapezius, infraspinatus in the fatigued limb, and serratus anterior in the both limbs with a fixed 2.5-cm spacing parallel to the muscle fiber. According to previous studies, the electrode locations for the anterior and middle deltoid were defined as $4 \mathrm{~cm}$ below the distal clavicle $\mathrm{e}^{23}$ and the halfway point between the acromion of the scapula and the deltoid tuberosity of the humerus, ${ }^{9}$ respectively; those for the upper and lower trapezius were defined as the halfway point between the spinous process of the seventh cervical vertebra and the acromion of the 
Scapular kinematics and serratus anterior fatigue

171 scapula ${ }^{23}$ and the halfway point between the spinous process of the seventh thoracic vertebra

172 and the trigonum scapula, ${ }^{9}$ respectively; that for the infraspinatus was defined as the halfway

173 point between the inferior angle of the scapula and the middle point between the acromion and

174 the trigonum scapula; ${ }^{18}$ and that for the serratus anterior was defined as the halfway point

175 between the leading edge of the latissimus dorsi and the trailing edge of the pectoralis major on

176 the seventh rib $^{15}$ (Figure 2).

the first 3 seconds of every 10 seconds up to 53 seconds (i.e. $0-3$ seconds; $10-13$ seconds; 20

flexed arm position without deviating their posture. The EMG signal of the maximal voluntary

previous studies. ${ }^{1,4}$ The raw EMG signals were processed using a bandpass filter and the root was normalized by the MVC of each muscle and the muscle activity was represented as 
Scapular kinematics and serratus anterior fatigue

190

191

193

Three-dimensional motion of the scapula and the humerus was measured during the muscle endurance test using a 6-df electromagnetic tracking device (Liberty, Polhemus, Colchester, VT, USA) at $120 \mathrm{~Hz}$ in the fatigued limb. This system consists of a transmitter, five sensors, and a digitizing stylus operated by an electronic unit. The transmitter was fixed on a rigid wooden board $40 \mathrm{~cm}$ from the floor and $30 \mathrm{~cm}$ behind the subjects. An electromagnetic field generated by the transmitter was sensed by these sensors and the stylus. This electromagnetic field represented the global coordinate system, with the $\mathrm{X}$-axis pointing forward, Y-axis pointing upward, Z-axis pointing to the right, and origin located at the transmitter. Next, the sensors were attached to the bony landmarks of the subjects with tape. The thoracic sensor was placed on the sternum just inferior to the jugular notch, the humeral sensor was placed on the middle point of the humerus with a thermoplastic cuff, and the scapular sensor was placed on the flat surface of the acromion. Based on these sensor placement, the local coordinate system of the thorax, humerus, and scapula were established by digitizing each bony landmark.

All definitions of the local coordinate system agreed with the shoulder standardization proposal of the International Society of Biomechanics. ${ }^{43}$ The rotation of the distal coordinate system was described with respect to the proximal coordinate system according to the Euler angle of the International Society of Biomechanics. The kinematics of the scapula segment relative to the thorax segment around the Sy-axis was defined as internal (positive) and external (negative) rotation, that around the Sx-axis was defined as downward (positive) and upward 
Scapular kinematics and serratus anterior fatigue

(negative) rotation, that around the Sz-axis was defined as posterior (positive) and anterior

(negative) tilt, and that of the humerus segment relative to the thorax segment around the Hx-

211 axis was defined as elevation (positive) and depression (negative) based on the shoulder

from 0 to 50 seconds, and all participants maintained the flexed arm position without deviating

215 their posture.

\section{Data analysis}

218 The statistical analysis was performed using SPSS Statistical software (version 22; IBM,

219 Armonk, NY, USA). Shapiro-Wilk test was used to confirm normality distribution. For the

220 muscle strength and endurance and the MDPF of the bilateral serratus anterior muscles, two-

221 way ANOVA with repeated measures on two factors (time [two levels, pre-fatigue; post-

222 fatigue]) $\times$ (limb [two levels, fatigue limb; control limb]) was used to examine the effect of

223 serratus anterior muscle fatigue on each parameter. When a significant interaction was found, a

224 paired $t$-test for normal distribution or Wilcoxon signed rank test for non-normal distribution

225 for post hoc analysis was performed to compare the pre- and post-fatigue values of each limb.

226 The MDPF values of all muscles except the serratus anterior were compared between the pre- 
Scapular kinematics and serratus anterior fatigue

muscles were fatigued due to the electric muscle stimulation. For the muscle activity and

scapular kinematics during the muscle endurance test, a two-way ANOVA with repeated measures of two factors (time [pre- and post-fatigue] $) \times($ seconds [six levels: $0,10,20,30,40$, hoc test was performed to compare the pre- and post-fatigue in each muscle. The significant main effect of seconds was ignored because the present study was interested in the comparison of pre- and post-fatigue. In addition, for the amount of change in the muscle activity calculated by subtracting pre-fatigue from post-fatigue in each muscle, a split-plot ANOVA with two factors (muscle [six levels: anterior deltoid, middle deltoid, upper trapezius, lower trapezius,

237 infraspinatus, serratus anterior]) $\times$ (seconds (six levels: 0, 10, 20, 30, 40, 50]) was used to determine which muscle was activated via serratus anterior fatigue though the muscle endurance test. When a significant main effect was found, a Tukey comparison of the post hoc 
Scapular kinematics and serratus anterior fatigue

\section{Results}

\section{Muscle strength and endurance}

The muscle strength and endurance results are shown in Table 1. For muscle strength, two-way ANOVA showed no significant interaction or main effects. For muscle endurance, two-way ANOVA showed a significant interaction between time and limb and a significant main effect of time. A post hoc test indicated that the muscle endurance significantly decreased in the fatigued limb after the fatigue task $(\mathrm{P}<.001)$.

\section{Muscle activity}

The muscle activity and degree of change in all muscles during the muscle endurance test are shown in Figure 4. For muscle activity, two-way ANOVA showed significant main effects of time in the SA, UT, and ISP, while the muscle activity significantly increased after the fatigue task. For amount of change in muscle activity, two-way ANOVA showed a significant main effect of muscle $(\mathrm{F}=7.00, \mathrm{P}<.001)$. The post hoc test indicated that the amount of change in the muscle activity of the upper trapezius was significantly greater than that of the other muscles $(\mathrm{P}<.001$ in all tests).

The MDPF values of the bilateral serratus anterior muscles and the other muscles are shown in Tables 1 and 2, respectively. For the MDPF of the serratus anterior, ANOVA showed a significant interaction, and then Wilcoxon signed rank test indicated a significantly decreased 
Scapular kinematics and serratus anterior fatigue

MDPF in the fatigued limb after the fatigue task $(\mathrm{P}<.001)$. The paired $t$-test indicated that the

263 MDPF of the upper trapezius significantly increased only in the fatigue task.

265 Scapular movement

266 Scapular movement before versus after the fatigue task is shown in Figure 5. Two-way ANOVA

267 showed no significant interaction and main effects in upward/downward rotation and

268 posterior/anterior tilt. For internal/external roation, there was no significant interaction $(\mathrm{F}=$

$2690.008, \mathrm{P}=.99)$; there was no significant main effect of seconds $(\mathrm{F}=0.68, \mathrm{P}=.64)$ but a

270 significant effect of time $(\mathrm{F}=5.87, \mathrm{P}=.02)$. The post hoc test indicated that the external rotation

271 after the fatigue task was significantly greater than that before it at all seconds $(\mathrm{P}=.02)$. 
Scapular kinematics and serratus anterior fatigue

\section{Discussion}

The present study investigated the effect of selective serratus anterior fatigue on muscle strength and endurance, scapular movement, and muscle activity at the flexed shoulder position. The results indicated no change in muscle strength but a significant change in muscle endurance.

Additionally, scapular movement and shoulder muscle activity were influenced by serratus anterior muscle fatigue. However, our hypothesis that the increase in scapular upward rotation was caused by compensatory activation of the upper trapezius muscle due to serratus anterior fatigue was rejected because upper trapezius, infraspinatus, and serratus anterior muscle activities were increased and the external rotation of the scapula was altered after the fatigue task. To our knowledge, this is the first study to demonstrate the changes in muscle endurance, scapular kinematics, and muscle activity following selective fatigue of the serratus anterior muscle. muscle fatigue was confirmed electromyographically via measurement of MDPF. A previous study indicated that the decline in MDPF was a sign of the physiological change in the muscle due to fatigue such as the slowing of muscle fiber conduction velocity, synchronization of motor units, and/or decreased firing frequency. ${ }^{40}$ Here we stimulated the serratus anterior muscle using a musculoskeletal electric stimulator, so the fatigue of the selective serratus anterior muscle could have been caused by the electric muscle stimulation because the decreased MDPF of the 
Scapular kinematics and serratus anterior fatigue

292

serratus anterior only occurred in the fatigued limb after the fatigue task.

Theoretically, muscle flexion strength of the serratus anterior muscle at the flexed shoulder position decreases after the fatigue because the serratus anterior muscle contributes to the upward rotation of the scapula during arm elevation ${ }^{28}$ and is maximally activated at shoulder flexion of $90-130^{\circ},{ }^{1}$ which is similar to the position used to measure the muscle strength of shoulder flexion in this study. However, muscle strength did not change after the fatigue task in this study. The serratus anterior does not flex the glenohumeral joint; rather, it stabilizes the scapula in the scapulothoracic joint due to its origin and insertion from the first to eighth or ninth ribs to the medial border of the scapula. Since the upper and lower trapezius muscles also stabilize the scapula, they may have compensated for the serratus anterior muscle's inability to stabilize the scapula; resulting no change in muscle strength was seen.

The present study showed that endurance decreased after serratus anterior muscle fatigue and that the upper trapezius, serratus anterior, and infraspinatus muscles were activated at the flexed shoulder position, which is inconsistent with our hypothesis. For the upper trapezius muscle, Ludewig and Cook $^{27}$ reported an increase in the muscle activity of the upper trapezius and a decrease in that of the serratus anterior muscle in the patient with shoulder impingement syndrome. Considering this report, it is possible that the upper trapezius muscles were activated to compensate for the functional impairment of the serratus anterior. For the serratus anterior muscle, greater muscle activity after the fatigue task is characteristic of muscle 
Scapular kinematics and serratus anterior fatigue

311 fatigue, the regulation of motor unit recruitment and rate coding patterns. ${ }^{34}$ The increased activity of the serratus anterior muscle after the fatigue task in this study is in accordance with this phenomenon. It is unknown how the mechanism to activate the infraspinatus muscle occurs after serratus anterior fatigue. Further research to investigate the interaction between muscles after fatigue is needed. increased after the fatigue task due to the change in the activities of the upper trapezius, serratus anterior, and infraspinatus muscles. In addition, the amount of change in the upper trapezius muscle activity was significantly greater than those of the other muscles. Moreover, the MDPF of the upper trapezius muscle increased significantly after the fatigue task. The upper trapezius retracts and upwardly rotates the scapula ${ }^{13}$, and the retraction corresponds to external rotation of the scapula in this study. Given the contribution of the upper trapezius muscle to the scapular movement, the increase in scapular external rotation that occurred in this study was a result of compensation of the upper trapezius for the functional impairment of the serratus anterior, causing so-called scapular winging. ${ }^{42}$ Scapular winging, in which the scapula rotates downward at rest and its inferior border becomes more prominent, corresponds to the scapular internal rotation in this study. Contrary to our hypothesis, no significant difference in upward scapular rotation angle was seen before versus after the fatigue task, which may be a result of compensation by the upper trapezius to avoid downward scapular rotation. 
Scapular kinematics and serratus anterior fatigue

factors in shoulder management. The excessive activation of the upper trapezius combined with

the decreased activation of the lower trapezius and the serratus anterior has been proposed to

be a contributor to abnormal scapular movement. ${ }^{10,11,27}$ Subjects with shoulder impingement

syndrome or shoulder pain typically present with excessive upper trapezius activity, attenuation

of the lower trapezius and serratus anterior activity ${ }^{17,26}$ and the scapular dysfunction at the same

time. ${ }^{17}$ In the current study, however, sole increase in upper trapezius muscle activation to

compensate for the fatigue of the serratus anterior without the decrease in the lower trapezius

muscle activation induced external rotation of the scapula which is similar to the impingement-

sparing change. These findings suggest that single muscle dysfunction could induce alteration

and identify not only the secondarily occurring compensation but also the primary muscle

dysfunction.

The present study investigated the acute effect of selective serratus anterior fatigue on

344 shoulder muscle activity and scapular movement to increase our knowledge of shoulder

biomechanics. However, it has some limitations. First, scapular kinematics up to $120^{\circ}$ were

analyzed in this study because the previous study ensured adequate reliability and validity of 
Scapular kinematics and serratus anterior fatigue

349 were all healthy men. Therefore, it is unclear whether our findings can be generalized to

350 selective cases of shoulder disease. Third, the degree of fatigue might not have been equal

351 among subjects, although the same duration of electrical muscle stimulation (i.e., 20 minutes)

352 was used. This may affect the results to some extent. Fourth, the fatigue induced by electrical

353 muscle stimulation would differ from the fatigue in a clinical situations to some extent. Fatigue

354 is classified into central fatigue, which occurs at central nervous system and involves central

355 activation failure, ${ }^{3,31}$ and peripheral fatigue, which occurs at the intramuscular contractile

356 machinery and involves metabolic inhibition of the contractile process and excitation-

357 contraction coupling failure. ${ }^{2,16,21}$ The fatigue in the current study is mainly accounted for with

358 peripheral fatigue. In other words, the current results could precisely show compensation 
Scapular kinematics and serratus anterior fatigue

\section{Conclusion}

362 The present study showed no changes in muscle strength but decreased muscle endurance after

363 selective fatigue of the serratus anterior. Increased muscle activities of the upper trapezius,

364 infraspinatus, and serratus anterior and external rotation of scapula were noted after the fatigue

365 task at the flexed shoulder position. These findings suggest that the compensatory motion to

366 avoid the internal scapular rotation occurred due to the increased shoulder muscle activity after

367 the fatigue task. 
Scapular kinematics and serratus anterior fatigue

369

370

\section{References}

1. Bandholm T, Rasmussen L, Aagaard P, Jensen BR, Diederichsen L. Force steadiness, muscle activity, and maximal muscle strength in subjects with subacromial impingement syndrome. Muscle Nerve 2006;34:631-639. doi:10.1002/mus.20636

2. Baker AL, Kostov KG, Miller RG, Weiner MW. Slow force recovery after longduration exercise: metabolic and activation factors in muscle fatigue. J Appl Physiol 1993;74:2294-2300.

3. Bigland-Ritchie B, Jones DA, Hosking GP, Edwards RH. Central and peripheral fatigue in sustained maximum voluntary contractions of human quadriceps muscle. Clin Sci Mol Med 1978;54:609-614.

4. Boettcher CE, Ginn KA, Cathers I. Which is the optimal exercise to strengthen supraspinatus? Med. Sci. Sports Exerc 2009;41:1979-1983. doi:10.1249/MSS.0b013e3181a740a7

5. Borstad JD, Szucs K, Navalgund A. Scapula kinematic alterations following a modified push-up plus task. Hum. Mov. Sci 2009;28:738-751. doi:10.1016/j.humov.2009.05.002

6. Chaubet V, Maitre J, Cormery B, Paillard T. Stimulated and voluntary fatiguing contractions of quadriceps femoris similarly disturb postural control in the bipedal stance. EurJ Appl Physiol. 2012;112:1881-1887. doi:10.1007/s00421-011-2168-9

7. Chopp JN, Fischer SL, Dickerson CR. The specificity of fatiguing protocols affects 
Scapular kinematics and serratus anterior fatigue

388

389

390

391

392

393

394

395

396

397

398

399

400

401

402

403

404

405

406

scapular orientation: Implications for subacromial impingement. Clin. Biomech.

(Bristol, Avon) 2011;26:40-45. doi:10.1016/j.clinbiomech.2010.09.001

8. Cook KF, Roddey TS, Gartsman GM, Olson SL. Development and psychometric evaluation of the Flexilevel Scale of Shoulder Function. Med. Care 2003;41:823-835. doi:10.1097/01.MLR.0000068540.82233.A8

9. Cools AM, Witvrouw EE, Declercq GA, Danneels LA, Cambier DC. Scapular muscle recruitment patterns: trapezius muscle latency with and without impingement symptoms. Am. J. Sports Med 2003;31:542-549. doi:10.1177/03635465030310041101

10. Cools AM, Declercq GA, Cambier DC, Mahieu NN, Witvrouw EE. Trapezius activity and intramuscular balance during isokinetic exercise in overhead athletes with impingement symptoms. Scand. J. Med. Sci. Sports 2007;17:25-33.

doi:10.1111/j.1600-0838.2006.00570.x

11. Cools AM1, Dewitte V, Lanszweert F, Notebaert D, Roets A, Soetens B, et al.

Rehabilitation of scapular muscle balance: which exercises to prescribe? Am J Sports

Med. 2007;35:1744-1751. doi:10.1177/0363546507303560

12. Cools AM, Struyf F, De Mey K, Maenhout A, Castelein B, Cagnie B. Rehabilitation of scapular dyskinesis: from the office worker to the elite overhead athlete. Br J Sports Med. 2014;48:692-697. doi:10.1136/bjsports-2013-092148

13. De Mey K, Danneels L, Cagnie B, Van den Bosch L, Flier J, Cools AM. Kinetic chain 
Scapular kinematics and serratus anterior fatigue

influences on upper and lower trapezius muscle activation during eight variations of a scapular retraction exercise in overhead athletes. J. Sci. Med. Sport 2013;16:65-70. doi:10.1016/j.jsams.2012.04.008

14. Ebaugh DD, McClure PW, Karduna AR. Effects of shoulder muscle fatigue caused by repetitive overhead activities on scapulothoracic and glenohumeral kinematics. J. Electromyogr. Kinesiol 2006;16:224-235. doi:10.1016/j.jelekin.2005.06.015

15. Ekstrom RA, Bifulco KM, Lopau CJ, Andersen CF, Gough JR. Comparing the function of the upper and lower parts of the serratus anterior muscle using surface electromyography. J. Orthop. Sports Phys. Ther 2004;34:235-243. doi:10.2519/jospt.2004.34.5.235

16. Fitts RH. Cellular mechanisms of mucle fatigue. Physiol Rev 1994;74:49-94.

17. Huang TS, Ou HL, Huang CY, Lin JJ. Specific kinematics and associated muscle activation in individuals with scapular dyskinesis. J Shoulder Elbow Surg 2015;24:1227-1237. doi:10.1016/j.jse.2014.12.022

18. Jaggi A, Malone AA, Cowan J, Lambert S, Bayley I, Cairns MC. Prospective blinded comparison of surface versus wire electromyographic analysis of muscle recruitment in shoulder instability. Physiother. Res. Int 2009;14:17-29. doi:10.1002/pri.407

19. Kamkar A, Irrgang JJ, Whitney SL. Nonoperative management of secondary shoulder impingement syndrome. J. Orthop. Sports Phys. Ther 1993;17:212-224. 
Scapular kinematics and serratus anterior fatigue

doi:10.2519/jospt.1993.17.5.212

20. Karduna AR, McClure PW, Michener LA, Sennett B. Dynamic measurements of threedimensional scapular kinematics: a validation study. J. Biomech. Eng 2001;123:184190. doi:10.1115/1.1351892

21. Kent-Braun JA. Central and peripheral contributions to muscle fatigue in humans during sustained maximal effort. Eur J Appl Physiol 1999;80:57-63. doi:10.1007/s004210050558

22. Kibler W Ben, McMullen J. Scapular dyskinesis and its relation to shoulder pain. J. Am. Acad. Orthop. Surg 2003;11:142-151.

23. Kibler W Ben, Sciascia AD, Uhl TL, Tambay N, Cunningham T. Electromyographic analysis of specific exercises for scapular control in early phases of shoulder rehabilitation. Am. J. Sports Med 2008;36:1789-1798.

doi:10.1177/0363546508316281

24. Kibler W Ben, Ludewig PM, McClure PW, Michener LA, Sciascia AD. Clinical implications of scapular dyskinesis in shoulder injury: the 2013 consensus statement from the 'Scapular Summit'. Br J Sports Med 2013;47:877-875. doi:10.1136/bjsports2013-092425

25. Landis JR, Koch GG. The measurement of ovserver agreement for categorical data. Biometrics 1997;33:159-174. 
Scapular kinematics and serratus anterior fatigue

26. Lin JJ, Hsieh SC, Cheng WC, Chen WC, Lai Y. Adaptive patterns of movement during arm elevation test in patients with shoulder impingement syndrome. J Orthop Res 2011;29:653-657. doi:10.1002/jor.21300

27. Ludewig PM, Cook TM. Alterations in shoulder kinematics and associated muscle activity in people with symptoms of shoulder impingement. Phys. Ther 2000;80:276291.

28. Ludewig PM, Cook TM, Nawoczenski DA. Three-dimensional scapular orientation and muscle activity at selected positions of humeral elevation. J. Orthop. Sports Phys. Ther 1996;24:57-65

29. Lukasiewicza C, McClure P, Michener L, Pratt N, Sennett B. Comparison of 3dimensional scapular position and orientation between subjects with and without shoulder impingement. J. Orthop. Sports Phys. Ther 1999;29:574-586

30. McClure PW, Michener LA, Sennett BJ, Karduna AR. Direct 3-dimensional measurement of scapular kinematics during dynamic movements in vivo. J. Shoulder Elbow Surg 2001;10:269-277

31. McKenzie DK, Bigland-Ritchie B, Gorman RB, Gandevia SC. Central and peripheral fatigue of human diaphragm and limb muscles assessed by twitch interpolation. $\mathrm{J}$ Physiol 1992;454:463-656

32. McQuade KJ, Dawson J, Smidt GL. Scapulothoracic muscle fatigue associated with 
Scapular kinematics and serratus anterior fatigue

464

465

alterations in scapulohumeral rhythm kinematics during maximum resistive shoulder elevation. J. Orthop. Sports Phys. Ther 1998;28:74-80

33. Maenhout A, DhoogeF, Van Herzeele M, Palman T, Cools A. Acromiohumeral distance and 3-dimentional scapular position change after overhead muscle fatigue. J Athl Train 2015;50:281-283. doi:10.4085/1062-6050-49.3.92

34. Moritani T, Sherman WM, Shibata M, Matsumoto T, Shinohara M. Oxygen availability and motor unit activity in humans. Eur. J. Appl. Physiol. Occup. Physiol 1992;64:552-556.

35. Paillard T, Maitre J, Chaubet V, Borel L. Stimulated and voluntary fatiguing contractions of quadriceps femoris differently disturb postural control. Neurosci Lett. 2010;477:48-51. dio:10.1016/j.neulet.2010.04.034

36. Paine RM, Voight M. The role of the scapula. J. Orthop. Sports Phys. Ther 1993;18:386-391

37. Paletta GA, Warner JJP, Warren RF, Deutsch A, Altchek DW. Shoulder kinematics with two-plane x-ray evaluation in patients with anterior instability or rotator cuff tearing. J. Shoulder Elbow Surg 1997;6:516-527

38. Ponce A, Fouque F, Cahouët V, Martin A. Effect of plantar flexor muscle fatigue induced by electromyostimulation on postural coordination. Neurosci Lett 2007;414:16-20. doi:10.1016/j.neulet.2006.10.025 
Scapular kinematics and serratus anterior fatigue

483

484

485

486

487

488

489

490

491

492

493

494

495

496

497

498

499

500

501

39. Shimo S, Sakamoto Y, Amari T, Gemma S. Validation of the shoulder36 for the activities of daily living with shoulder disorders. J. Phys. Ther. Sci 2017;29:635-640. doi:10.1589/jpts.29.635

40. Vestergaard-Poulsen P, Thomsen C, Sinkjaer T, Stubgaard M, Rosenfalck A, Henriksen O. Simultaneous electromyography and 31P nuclear magnetic resonance spectroscopy--with application to muscle fatigue. Electroencephalogr. Clin. Neurophysiol 1992;85:402-411.

41. Warner JJ, Micheli LJ, Arslanian LE, Kennedy J, Kennedy R. Scapulothoracic motion in normal shoulders and shoulders with glenohumeral instability and impingement syndrome. A study using Moiré topographic analysis. Clin. Orthop. Relat. Res 1992;285:191-199.

42. Watson CJ, Schenkman M. Physical therapy management of isolated serratus anterior muscle paralysis. Phys. Ther 1995;75:194-202.

43. Wu G, van der Helm FCT, (DirkJan) Veeger HEJ, Makhsous M, Van Roy P, Anglin C, et al. ISB recommendation on definitions of joint coordinate systems of various joints for the reporting of human joint motion-Part II: shoulder, elbow, wrist and hand. J. Biomech 2005;38:981-992. doi:10.1016/j.jbiomech.2004.05.042 
Scapular kinematics and serratus anterior fatigue

502

503

504

505

506

507

508

509

510

511

512

513

514

515

516

517

518

519

520

\section{Figure and Table Legends}

Figure 1. Measurement posture of the muscle endurance test. Participants sat and flexed the shoulder $90^{\circ}$, fully extended the elbow, and kept the trunk upright while holding a dumbbell corresponding to $40 \%$ of the muscle strength as long as possible.

Figure 2. Locations of EMG electrodes for each muscle. AD, anterior deltoid muscle; MD, middle deltoid muscle; UT, upper trapezius muscle; LT, lower trapezius muscle, ISP, infraspinatus muscle; SA, serratus anterior muscle.

Figure 3. Definition of the coodinate system and motions relative to the thorax for the scapula and humerus. In the local coordinate system of the scapula, the Sx axis was perpendicular to the plane defined by the TS, AA, and AI; Sy-axis was defined as the cross product of the Sxaxis and Sz-axis; and Sz-axis was directed from the TS to the AA. In the local coordinate system of the humerus, the Hx-axis was perpendicular to the plane defined by the GH, $\mathrm{LH}$, and $\mathrm{MH}$. TS, trigoum spina scapula; AA, acromial angle; AI, inferior angle; GH, glenohumeral rotation center; EL, lateral epicondyle; EM, medial epicondyle; ER, external rotation, IR, internal rotation; UR, upward rotation; DR, downward rotation; AT anterior tilt; PT, posterior tilt.

Figure 4. Muscle activity and amount of change of muscle activity at the flexed shoulder 
Scapular kinematics and serratus anterior fatigue

521

522 Table 2. MDPF of all muscles but the serratus anterior before versus after the fatigue task.

position. AD, anterior deltoid (A); MD, middle deltoid (B); UT, upper trapezius (C); LT, lower trapezius (D); ISP, infraspinatus (E); SA, serratus anterior (F); Change in muscle activities (G); Pre, the value of pre-fatigue task; Post, the value of post fatigue task. The asterisk represents the significant main effect and indicated that the value of Post is significantly greater than that of Pre. The dagger represents the significant main effect and indicates that the changes in UT was significantly greater than that of other muscles.

Figure 5. Scapular kinematics at the flexed shoulder position. Left, internal/external rotation of the scapula; middle, downward/upward rotation of the scapula; right, posterior/anterior tilt. The solid line and the dotted line represent the values of the scapula in pre- and post-fatigue, respectively. The asterisk indicates the significant main effect of the period and that the external rotation after the fatigue task was significantly greater than that before it.

\section{Table 1. Muscle strength and endurance and MDPF of the serratus anterior before versus after} the fatigue task. 
Scapular kinematics and serratus anterior fatigue

$539 \quad$ Figure 1

540

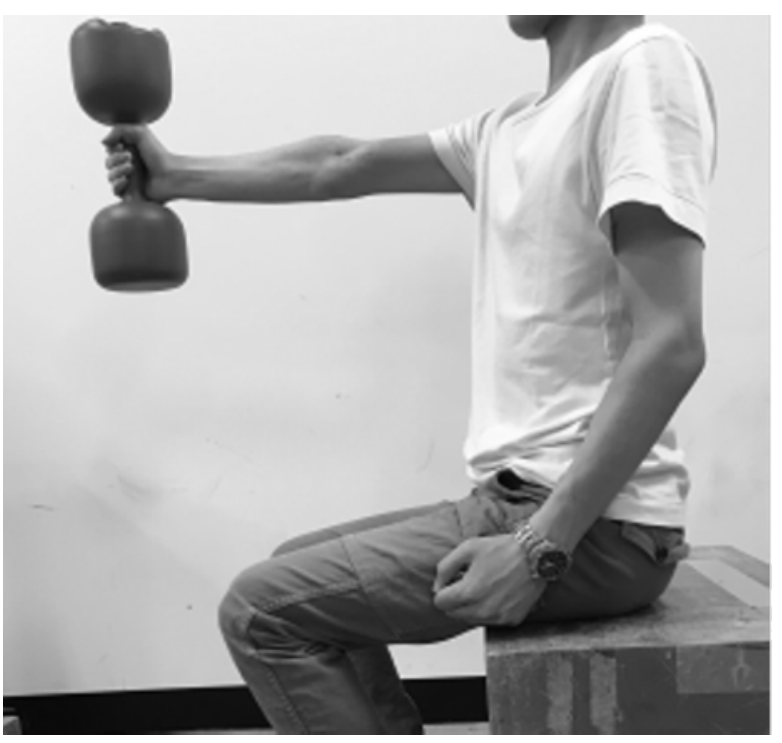

541 
Scapular kinematics and serratus anterior fatigue

Figure 2

543

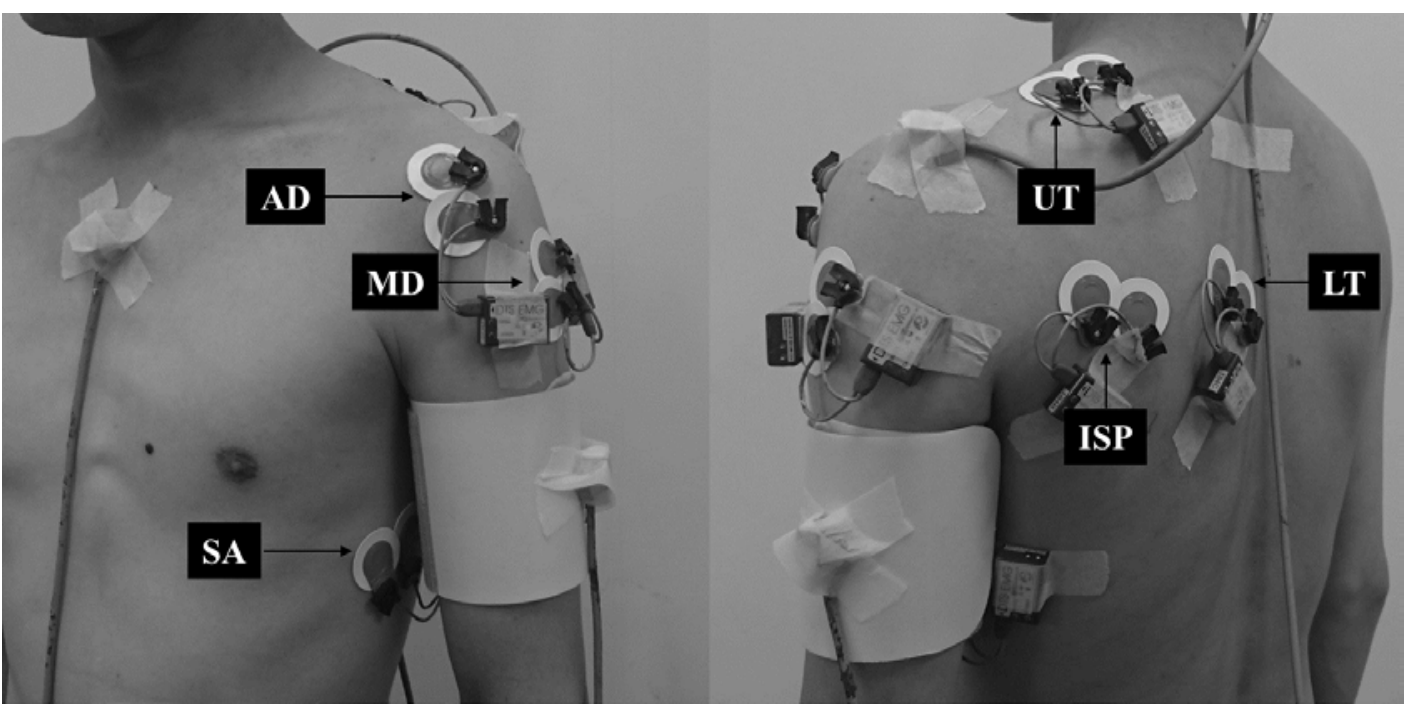

544 
Scapular kinematics and serratus anterior fatigue

Figure 3

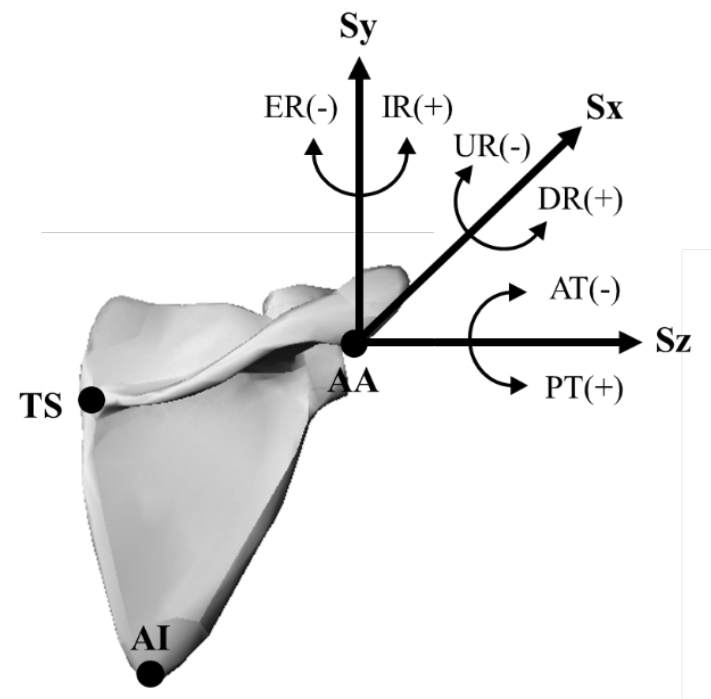

Scapula

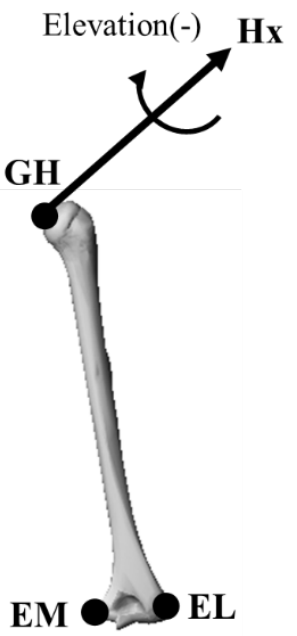

Humerus 
Scapular kinematics and serratus anterior fatigue

Figure 4
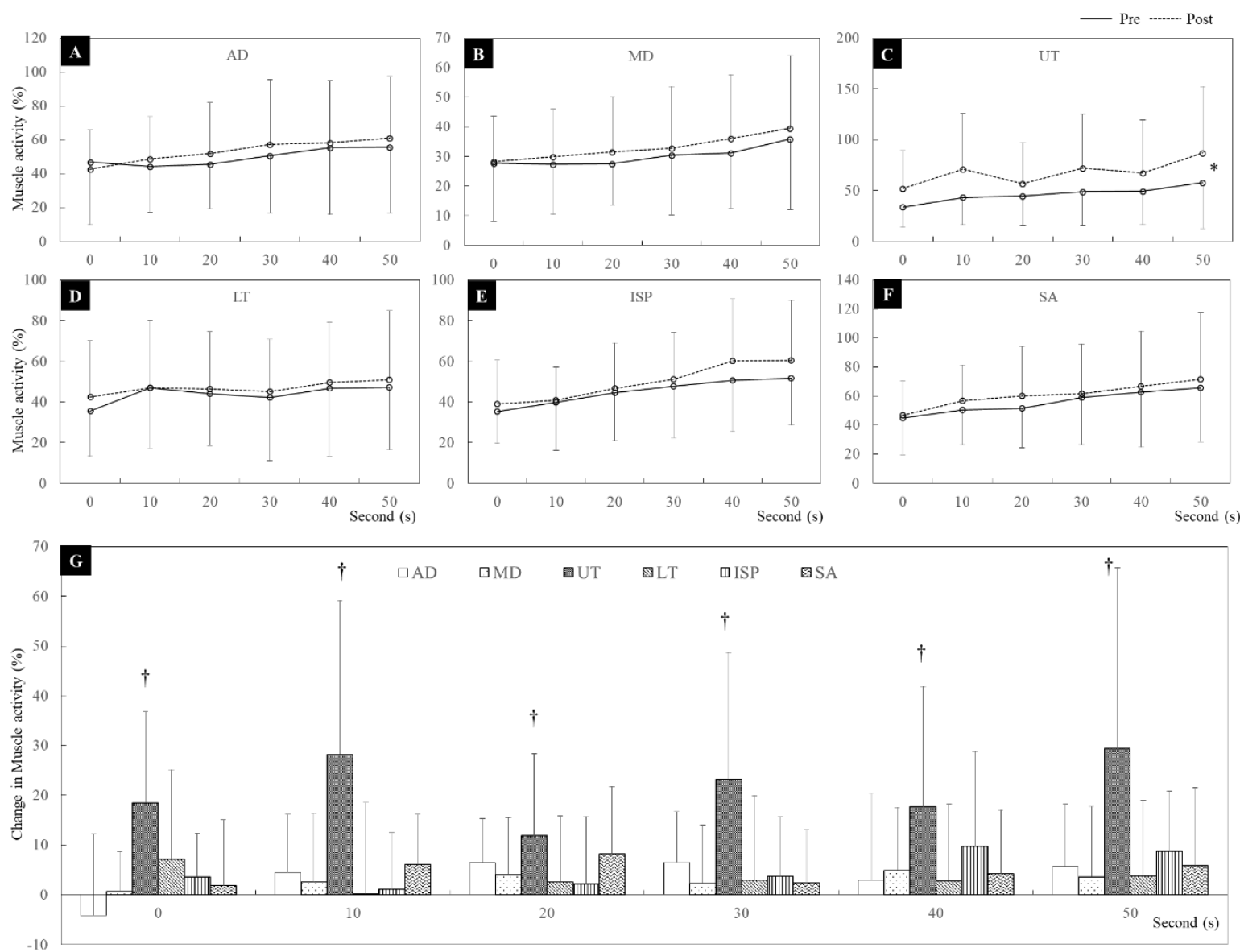
Scapular kinematics and serratus anterior fatigue

551

\section{Figure 5}

552
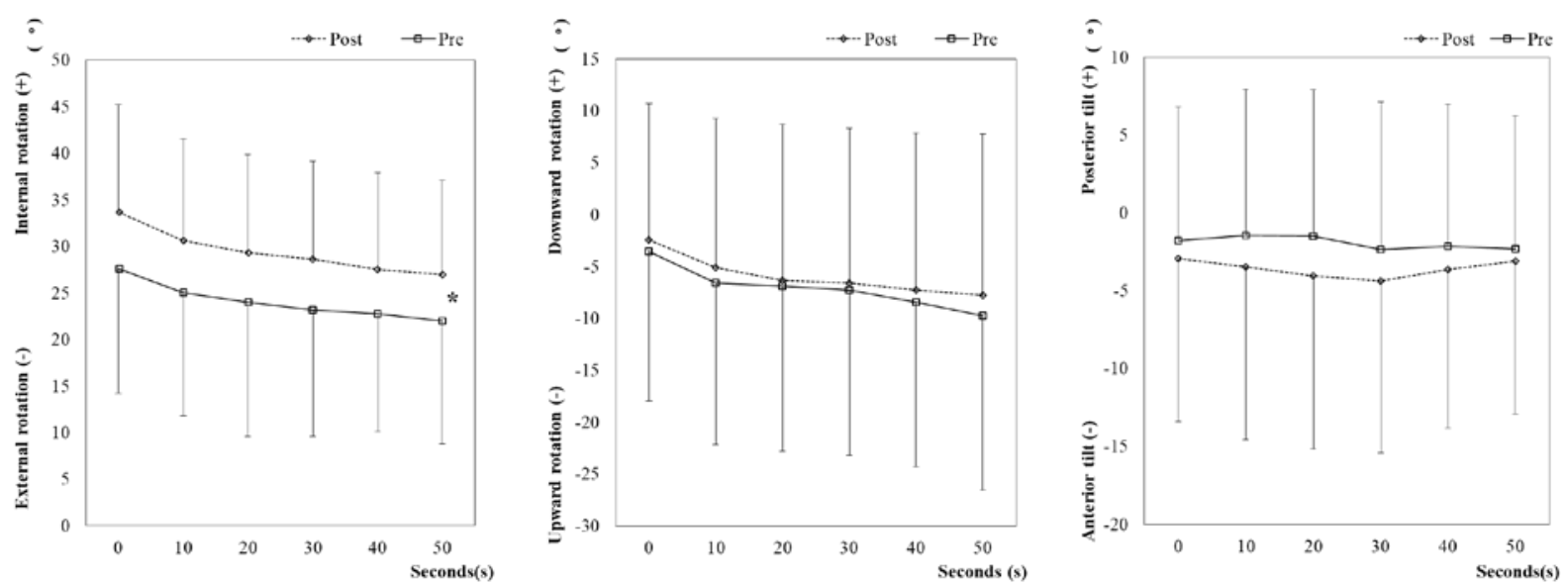

553 
Scapular kinematics and serratus anterior fatigue

Table 1

\begin{tabular}{|c|c|c|c|c|c|c|}
\hline & \multicolumn{2}{|c|}{ Muscle strength (Nm) } & \multicolumn{2}{|c|}{ Muscle endurance (sec) } & \multicolumn{2}{|c|}{ MDPF of serratus anterior } \\
\hline & Fatigue & Control & Fatigue & Control & Fatigue & Control \\
\hline Pre & $57.5 \pm 13.5$ & $58.3 \pm 10.8$ & $80.7 \pm 13.0$ & $77.0 \pm 17.1$ & $59.8 \pm 7.2$ & $59.0 \pm 10.8$ \\
\hline Post & $58.2 \pm 12.2$ & $57.6 \pm 11.6$ & $66.5 \pm 15.3^{*}$ & $76.7 \pm 16.7$ & $51.2 \pm 6.0^{*}$ & $59.1 \pm 11.0$ \\
\hline Interaction & \multicolumn{2}{|c|}{$F=1.08, P=.32$} & \multicolumn{2}{|c|}{$\mathrm{F}=16.91, \mathrm{P}<.001$} & \multicolumn{2}{|c|}{$\mathrm{F}=41.92, \mathrm{P}<.001$} \\
\hline & \multicolumn{2}{|c|}{ Period: $\mathrm{F}<0.01, \mathrm{P}=.97$} & \multicolumn{2}{|c|}{ Period: $\mathrm{F}=15.53, \mathrm{P}<.001$} & \multicolumn{2}{|c|}{ Period: $\mathrm{F}=73.72, \mathrm{P}<.001$} \\
\hline & \multicolumn{2}{|c|}{ Limb: $\mathrm{F}<0.01, \mathrm{P}=.98$} & \multicolumn{2}{|c|}{ Limb: $\mathrm{F}=0.78, \mathrm{P}=.42$} & \multicolumn{2}{|c|}{ Limb: $\mathrm{F}=2.92, \mathrm{P}=.11$} \\
\hline
\end{tabular}

555 Fatigue, fatigue limb; Control, control limb; Pre, the value of pre fatigue task; Post, the value 556 of post fatigue task. The asterisk indicates that the value of Post is significantly lower than that 557 of Pre.

558

559 
Scapular kinematics and serratus anterior fatigue

560 Table 2

\begin{tabular}{|c|c|c|c|c|c|}
\hline MDPF (Hz) & $\mathrm{AD}$ & MD & UT & LT & ISP \\
\hline Pre & $78.9 \pm 12.7$ & $68.5 \pm 10.5$ & $68.6 \pm 10.6$ & $52.1 \pm 9.9$ & $101.4 \pm 23.7$ \\
\hline Post & $80.3 \pm 12.8$ & $68.0 \pm 11.7$ & $71.3 \pm 11.6$ & $52.1 \pm 9.6$ & $101.7 \pm 21.6$ \\
\hline P value & $\mathrm{P}=.30$ & $\mathrm{P}=.69$ & $\mathrm{P}=.02$ & $\mathrm{P}=.99$ & $\mathrm{P}=.93$ \\
\hline
\end{tabular}

561 AD, anterior deltoid; MD, middle deltoid; UT, upper trapezius; LT, lower trapezius; ISP,

562 infraspinatus; Pre, the value of pre fatigue task; Post, the value of post fatigue task.

563

564 